\title{
COMPOSITION OPERATORS ON FINITE RANK MODEL SUBSPACES
}

\author{
JAVAD MASHREGHI \\ Département de mathématiques et de statistique, Université Laval, Québec G1K 7P4, Canada \\ e-mail: javad.mashreghi@mat.ulaval.ca \\ and MAHMOOD SHABANKHAH \\ Department of Mathematics and Statistics, McGill University, Montreal, Québec H3A 2K6, Canada \\ e-mail:mshaban@math.mcgill.ca
}

(Received 5 April 2011; accepted 16 March 2012; first published online 2 August 2012)

\begin{abstract}
We give a complete description of bounded composition operators on model subspaces $K_{B}$, where $B$ is a finite Blaschke product. In particular, if $B$ has at least one finite pole, we show that the collection of all bounded composition operators on $K_{B}$ has a group structure. Moreover, if $B$ has at least two distinct finite poles, this group is finite and cyclic.
\end{abstract}

2010 Mathematics Subject Classification. Primary: 47B33, Secondary: 30D50, $32 \mathrm{~A} 35$.

1. Introduction. Let $\Omega$ be a domain in the Riemann sphere $\overline{\mathbb{C}}$, and denote the family of all analytic functions $f: \Omega \longrightarrow \overline{\mathbb{C}}$ by $H(\Omega)$. Assume that a linear subspace $X \subset H(\Omega)$, endowed with a norm, is a Banach space. If $\varphi: \Omega \longrightarrow \Omega$ is analytic, then the mapping

$$
C_{\varphi} f=f \circ \varphi, \quad(f \in X),
$$

is called the composition operator with symbol $\varphi$. We are interested in finding symbols $\varphi$ for which $X$ is invariant under $C_{\varphi}$ and the restriction $C_{\varphi}: X \longrightarrow X$ is a bounded operator, i.e. $C_{\varphi} \in \mathcal{L}(X)$. The collection of all bounded composition operators will be denoted by $\mathcal{L}_{c}(X)$. It is easy to verify that $\mathcal{L}_{c}(X)$ has a semi-group structure and

$$
C_{\varphi} \circ C_{\psi}=C_{\psi \circ \varphi}
$$

The identity function $\varphi(z)=z$, which is simply denoted by $z$, induces the identity element $i d=C_{z}$ in $\mathcal{L}_{c}(X)$. We will use the notation $\varphi^{[n]}=\varphi \circ \cdots \circ \varphi, n$ times. In our discussion, we will see that quite often $\mathcal{L}_{c}(X)$ is precisely the trivial group $\left\{C_{z}\right\}$.

The classical subordination principle of Littlewood can be rephrased in this setting by saying that, for any symbol $\varphi: \mathbb{D} \longrightarrow \mathbb{D}$, the mapping

$$
\begin{aligned}
C_{\varphi}: H^{p}(\mathbb{D}) & \longrightarrow H^{p}(\mathbb{D}) \\
f & \longmapsto f \circ \varphi
\end{aligned}
$$

is a well-defined bounded operator on the classical Hardy space $H^{p}(\mathbb{D})$.

This work was supported by NSERC (Canada), NRS (France) and FQRNT (Québec). 
It is rather impossible to make justice and give a comprehensive list of papers on this subject. We just make a very short list of papers that we consulted in the preparation of this note: $[3,4]$ treat the composition operators on Hardy space $H^{2}$, [14] characterises the compact composition operators on $H^{2},[\mathbf{1 0}, \mathbf{1 1}]$ is for Begrman and weighted Hardy spaces, [15] for Besov spaces, [16] for BMO, [2, 7, 8] for Bloch spaces and finally [17] for the Dirichlet space. The compactness of $C_{\varphi}$ on Hardy spaces is also studied in [13]. There is also another characterisation for compactness in [9]. Composition operators on many other spaces have been extensively studied by many authors. The books in [5] and [13] provide an excellent exposition as well as a rich bibliography of the subject.

Let $\left(\lambda_{n}\right)_{1 \leq n \leq N}$ be $N$ distinct points in the punctuated open unit disc $\mathbb{D} \backslash\{0\}$, let $\left(m_{n}\right)_{0 \leq n \leq N}$ be a finite sequence of non-negative integers and let $\gamma$ be a unimodular constant. Then the special rational function

$$
B(z)=\gamma z^{m_{0}} \prod_{n=1}^{N}\left(\frac{\lambda_{n}-z}{1-\bar{\lambda}_{n} z}\right)^{m_{n}}
$$

is called a finite Blaschke product. Clearly, $B$ is an analytic function on $\overline{\mathbb{C}}$ with zeros of order $m_{n}$ at $\lambda_{n}$, and it fulfills the characterising property

$$
|B(\zeta)|=1, \quad(\zeta \in \mathbb{T})
$$

Based on the above definition, a unimodular constant should also be treated as a finite Blaschke product. However, to avoid certain technical difficulties, in the following we exclude this case and always assume that a finite Blaschke product has at least one zero.

The finite dimensional subspace spanned by the functions

$$
1, z, z^{2}, \ldots, z^{m_{0}-1}
$$

and

$$
\frac{1}{1-\bar{\lambda}_{n} z}, \frac{1}{\left(1-\bar{\lambda}_{n} z\right)^{2}}, \ldots, \frac{1}{\left(1-\bar{\lambda}_{n} z\right)^{m_{n}}}, \quad(1 \leq n \leq N),
$$

is called the model subspace generated by $B$ and is denoted by $K_{B}$. In other words, $f \in K_{B}$ if and only if there are some constants $c_{n, k}$ such that

$$
f(z)=\sum_{k=0}^{m_{0}-1} c_{0, k} z^{k}+\sum_{n=1}^{N} \sum_{k=1}^{m_{n}} \frac{c_{n, k}}{\left(1-\bar{\lambda}_{n} z\right)^{k}} .
$$

As a very special case of the well-known result of Beurling [1] on invariant subspaces of the shift operator $S$ on the Hardy space $H^{2}, K_{B}$ is the closed subspace $H^{2}$, which is generated by $S^{* k}, k \geq 0$. Owing to the special form of functions in $K_{B}$, we remind that

$$
k_{\lambda}(z)=\frac{1}{1-\bar{\lambda} z}
$$

is called the Cauchy kernel. As a matter of fact, $k_{\lambda}$ is the reproducing kernel of the Hardy-Hilbert space $H^{2}(\mathbb{D})$. 
In this paper, we completely characterise bounded composition operators on the model subspace $K_{B}$, which is generated by the finite Blaschke product $B$. Our characterisation reveals that the structure of $\mathcal{L}_{c}\left(K_{B}\right)$ is heavily glued to the distribution of zeros of $B$. Naively speaking, we can say that $\mathcal{L}_{c}\left(K_{B}\right)$ has a rich structure, provided that the zeros of $B$ have a rich geometrical symmetry. Due to lack of symmetry, we mostly have $\mathcal{L}_{c}\left(K_{B}\right)=\{i d\}$. Moreover, under certain mild conditions to ensure the symmetry, $\mathcal{L}_{c}\left(K_{B}\right)$ is a finite cyclic group. There are also some marginal cases that do not fall in the above two categories. These facts are crystallised in the next section.

Some precision is needed to describe the symbols that are discussed below. Back to the general setting which was pictured above, if we consider $K_{B}$ as a closed subspace of $H^{2}(\mathbb{D})$, we should treat its elements as analytic functions on $\mathbb{D}$. Hence, we seek symbols $\varphi: \mathbb{D} \longrightarrow \mathbb{D}$ for which $C_{\varphi}$ is a bounded operator on $K_{B}$. We will determine all such $\varphi s$ for a given $K_{B}$. However, the elements of $K_{B}$ live on $\overline{\mathbb{C}}$ and thus it is natural to look for symbols $\varphi: \overline{\mathbb{C}} \longrightarrow \overline{\mathbb{C}}$ that induce bounded operators on $K_{B}$. We will see that this class is larger and, as a matter of fact, includes all the previous symbols $\varphi: \mathbb{D} \longrightarrow \mathbb{D}$. It is well known that the only analytic functions on $\overline{\mathbb{C}}$ are rational functions. Therefore, we need to characterise all rational functions $\varphi$ such that $C_{\varphi}$ maps $K_{B}$ into itself. The boundedness then easily follows from Littlewood's theorem.

2. The structure of $\mathcal{L}_{c}\left(K_{B}\right)$. To understand the structure of $\mathcal{L}_{c}\left(K_{B}\right)$, on the one hand, we should distinguish between the cases where $B$ has no zero, a simple zero, or a multiple zero at the origin, and on the other hand, the case where $B$ has one or more distinct zeros other than the origin. Hence, totally we face six different situations. In the following, the set of zeros of $B$, multiplicity not counted, is denoted by $\mathcal{Z}(B)$. Therefore, when we write $\mathcal{Z}(B)=\{\lambda\}$, it does not mean that $\lambda$ is necessarily a simple zero, and when we write $\mathcal{Z}(B)=\left\{\lambda_{1}, \lambda_{2}, \ldots\right\}$, it implicitly implies that $\lambda_{1} \neq \lambda_{2}$.

2.1. $\mathcal{Z}(B)=\{\lambda\}, B(0) \neq 0$. In the first step, we study the Blaschke products with a single zero, other than the origin, which might be repeated a finite number of times.

THEOREM 2.1. Let

$$
B(z)=\left(\frac{\lambda-z}{1-\bar{\lambda} z}\right)^{n}, \quad(\lambda \neq 0, n \geq 1) .
$$

Then

$$
\mathcal{L}_{c}\left(K_{B}\right)=\left\{C_{(1-\bar{\lambda} a) z+a}: a \in \mathbb{C}, a \neq 1 / \bar{\lambda}\right\}
$$

Proof. Let $C_{\varphi} \in \mathcal{L}_{c}\left(K_{B}\right)$. Then we must have $C_{\varphi} k_{\lambda}^{n} \in K_{B}$. Therefore, by (1.3), there are some constants $c_{k}$ such that

$$
\left(C_{\varphi} k_{\lambda}^{n}\right)(z)=\frac{1}{(1-\bar{\lambda} \varphi(z))^{n}}=\sum_{k=1}^{n} \frac{c_{k}}{(1-\bar{\lambda} z)^{k}} .
$$


Firstly, this representation shows that $\varphi(z)=a+b z$, for some $a, b \in \mathbb{C}$. Secondly, since

$$
\left(C_{\varphi} k_{\lambda}\right)(z)=\frac{1}{1-\bar{\lambda} a} \times \frac{1}{1-\overline{\left(\frac{\bar{b} \lambda}{1-\bar{a} \lambda}\right)} z},
$$

we must have $1-\bar{\lambda} a \neq 0$ and

$$
\lambda=\frac{\bar{b} \lambda}{1-\bar{a} \lambda},
$$

or equivalently $b=1-\bar{\lambda} a$.

If we put $\varphi_{a}(z)=(1-\bar{\lambda} a) z+a$, then, by $(1.1)$,

$$
C_{\varphi_{a}} \circ C_{\varphi_{b}}=C_{\varphi_{b} \circ \varphi_{a}}=C_{\varphi_{a+b-\bar{\lambda} a b}} .
$$

Hence, $\mathcal{L}_{c}\left(K_{B}\right)$ is a group which is isomorphic to $\mathbb{C} \backslash\{1 / \bar{\lambda}\}$ endowed with the law of composition

$$
a * b=a+b-\bar{\lambda} a b .
$$

The identity element is zero and the inverse of $a$ is $-a /(1-\bar{\lambda} a)$.

If we seek only the symbols $\varphi: \mathbb{D} \longrightarrow \mathbb{D}$, we end up with the same characterisation accompanied by an extra condition

$$
|1-\bar{\lambda} a|+|a| \leq 1
$$

But, this inequality holds just for $a=0$, i.e. the only acceptable symbol is $\varphi(z)=z$.

2.2. $\mathcal{Z}(B)=\left\{\lambda_{1}, \lambda_{2}, \ldots\right\}, \quad B(0) \neq 0$. In the next step, we find bounded composition operators on $K_{B}$ when $B$ is not zero at the origin and has at least two distinct zeros. It is easy to see that none of the transformations provided in Theorem 2.1 (except of course $\varphi(z)=z$ ), which maps a reproducing kernel to itself, works in this situation. However, if zeros of $B$ follow a special pattern, we may have some acceptable symbols $\varphi$ such that $C_{\varphi}$ maps a reproducing kernel to another and thus be bounded on $K_{B}$.

LEMMA 2.2. Let $B$ be a finite Blaschke product with $B(0) \neq 0$, and with at least two distinct zeros. Let $\varphi$ be a rational function, $\varphi(z) \neq z$. Then $C_{\varphi}$ maps $K_{B}$ into itself if and only if

$$
\varphi(z)=a z+b
$$

and the following conditions are fulfilled:

(i) $a \in \mathbb{T} \backslash\{1\}$ is of finite order, i.e. there is an integer $n>1$ such that $a^{n}=1$.

(ii) If $\lambda$ is a zero of $B$, then so is $\eta(\lambda)$, where $\eta$ is the Möbius transformation

$$
\eta(z)=\frac{\bar{a} z}{1-\bar{b} z} .
$$

(iii) If $\lambda$ is a zero of $B$, then the zeros $\lambda, \eta(\lambda), \ldots, \eta^{[n-1]}(\lambda)$ have the same order. 
Proof. Pick any zero $\lambda_{\ell}$ of B. Then we must have $C_{\varphi} k_{\lambda_{\ell}} \in K_{B}$. Therefore, by (1.3), there are some constants $c_{n, k}$ such that

$$
\left(C_{\varphi} k_{\lambda_{\ell}}\right)(z)=\frac{1}{1-\bar{\lambda}_{\ell} \varphi(z)}=\sum_{n=1}^{N} \sum_{k=1}^{m_{n}} \frac{c_{n, k}}{\left(1-\bar{\lambda}_{n} z\right)^{k}} .
$$

If we write $\varphi=P / Q$, where $P$ and $Q$ are polynomials with no common divisor, then the above identity puts certain restrictions on the degrees of $P$ and $Q$. More precisely, we must have

$$
\operatorname{deg} Q<\operatorname{deg} P \leq N_{0},
$$

with $N_{0}=m_{1}+\cdots+m_{N}$ being the total number of zeros of $B$.

Now, we do the iteration. If $C_{\varphi}$ is bounded on $K_{B}$, so is $C_{\varphi^{[k]}}$. Hence, it follows that $\varphi^{[k]}$ must also be a rational function with the same restrictions on the degrees of its numerator and denominators as above. It is easy to see that the degree of numerator of $\varphi^{[k]}$ is equal to $(\operatorname{deg} P)^{k}$. Thus, we must have $\operatorname{deg} P=1$ and $\operatorname{deg} Q=0$, which means that $\varphi(z)=a z+b$, for some $a, b \in \mathbb{C}$.

Let $\lambda$ be any zero of $B$. Then

$$
\left(C_{\varphi} k_{\lambda}\right)(z)=\frac{1}{1-\bar{\lambda}(a z+b)}=\frac{1}{1-\bar{\lambda} b} \cdot \frac{1}{1-\overline{\eta(\lambda)} z} .
$$

Hence, we conclude that $\eta(\lambda)$ must also be a zero of $B$ and clearly $\eta(\lambda) \neq 0$. Since we have assumed that $B$ has at least two distinct zeros, at this step we are not forced to assume that $\eta(\lambda)=\lambda$. In fact, repeating this process shows that, for each $k \geq 1, \eta^{[k]}(\lambda)$ is a zero of $B$. With this observation, we can say more about the coefficients $a$ and $b$. Incidentally, the above calculation also shows that

$$
\left(C_{\varphi} k_{\lambda}^{d}\right)(z)=\frac{1}{(1-\bar{\lambda} b)^{d}} \cdot \frac{1}{(1-\overline{\eta(\lambda)} z)^{d}} .
$$

Therefore, if $\lambda$ is a zero of order $d$, then the order of $\eta(\lambda)$ is at least $d$. By the periodicity property (see below), we conclude that they have actually the same order.

If $a \notin \mathbb{T}$, then $\eta$ is a loxodromic Möbius transformation and thus the iterates of a point that is not a fixed point (in our case $\lambda$ ) form an infinite sequence that converge to one of the absorbing fixed points of $\eta$. This is a contradiction.

If $a=1$ and $b \neq 0$, then $\eta$ is parabolic and the situation is similar to the previous case. As a matter of fact, in this situation, $\eta^{[k]}(\lambda), k \geq 1$, is a sequence of distinct points that converges to zero.

If $a=1$ and $b=0$, then $\varphi(z)=z$, which is acceptable in any situation.

If $a \in \mathbb{T} \backslash\{1\}$, then $\eta$ is elliptic. This is an interesting case. Since we are dealing with the finite Blaschke products, the sequence $\eta^{[k]}(\lambda), k \geq 1$ has to be periodic. This happens if and only if $\eta$ is of finite order. But we can write $\eta=T^{-1} \circ \rho \circ T$, where $\rho(z)=\bar{a} z$ and $T(z)=z /(\alpha z+\beta)$, where $\alpha$ and $\beta$ are constants such that $\alpha(\bar{a}-1)=\beta \bar{b}$. Therefore, $\eta$ is of finite order if and only if so is $a$.

For a detailed discussion of fixed points of the Möbius transformation, see [12, Chapter 3, VII] and [13, Chapter 0].

In Theorem 2.1, we completely characterised $\mathcal{L}_{c}\left(K_{B}\right)$. But Lemma 2.2 has a slightly different nature. More precisely, the constants $a$ and $b$ were not fully specified. We will 
discuss further on this issue in Section 3. Nevertheless, we can still say something about the algebraic structure of $\mathcal{L}_{c}\left(K_{B}\right)$. The following result reveals that $\mathcal{L}_{c}\left(K_{B}\right)$ is a finite cyclic group.

THEOREM 2.3. Let $B$ be a finite Blaschke product with $B(0) \neq 0$, and with at least two distinct zeros. Then there exists an integer $n \geq 1$ and a linear function $\varphi(z)=a z+b$ such that $\varphi^{[n]}(z)=z$ and

$$
\mathcal{L}_{c}\left(K_{B}\right)=\left\{C_{z}, C_{\varphi}, C_{\varphi^{[2]}}, \ldots, C_{\varphi^{[n-1]}}\right\}
$$

Proof. If $\varphi(z)=a z+b$, then it is easy to see that

$$
\varphi^{[k]}(z)=a^{k} z+\left(a^{k-1}+a^{k-2}+\cdots+1\right) b, \quad(k \geq 1) .
$$

If $a^{n}=1$, but $a^{n-1} \neq 1$, we have $a^{n-1}+a^{n-2}+\cdots+1=0$. Therefore, $C_{\varphi}^{n}=C_{\varphi^{[n]}}=$ $C_{z}=i d$. Thus, by Lemma 2.2, we surely have the inclusion

$$
\left\{C_{z}, C_{\varphi}, C_{\varphi^{[2]}}, \ldots, C_{\varphi^{[n-1]}}\right\} \subset \mathcal{L}_{c}\left(K_{B}\right),
$$

and the set on the left side has $n$ distinct elements. In the same token,

$$
\eta^{[k]}(z)=\frac{\bar{a}^{k} z}{1-\bar{b}\left(1+\bar{a}+\cdots+\bar{a}^{k-1}\right) z}, \quad(k \geq 1),
$$

and thus $\eta^{[n]}=i d$, but $\eta^{[k]} \neq i d$ for $1 \leq k<n$. This means that if $\lambda$ is a zero of $B$, then $\lambda, \eta(\lambda), \ldots, \eta^{[n-1]}(\lambda)$ are distinct zeros of $B$, which have the same order. If we take $n$ to be the maximal integer with the above properties, then (2.2) turns to an equality.

Lemma 2.2 is a manifestation of the fact that $\mathcal{L}_{c}\left(K_{B}\right)$ has a rich structure provided that the zeros of $B$ follow a certain pattern. It also provides an algorithm to find $\mathcal{L}_{c}\left(K_{B}\right)$. To do so, we first pick all the zeros of order one and construct the Blaschke product $B_{1}$. Then we pick all the zeros of order two, without considering the repetition, and construct the Blaschke product $B_{2}$ etc. Then we have

$$
\mathcal{L}_{c}\left(K_{B}\right)=\mathcal{L}_{c}\left(K_{B_{1}}\right) \cap \mathcal{L}_{c}\left(K_{B_{2}}\right) \cap \cdots .
$$

For example, if $0<|\alpha|<|\beta|<1, M \geq 1, N \geq 1$, and

$$
B(z)=\prod_{n=1}^{6}\left(\frac{\alpha e^{i n \pi / 3}-z}{1-\bar{\alpha} e^{-i n \pi / 3} z}\right)^{M} \times \prod_{n=1}^{15}\left(\frac{\beta e^{i 2 n \pi / 15}-z}{1-\bar{\beta} e^{-i 2 n \pi / 15} z}\right)^{N},
$$

then we may define

$$
B_{M}(z)=\prod_{n=1}^{6} \frac{\alpha e^{i n \pi / 3}-z}{1-\bar{\alpha} e^{-i n \pi / 3} z} \quad \text { and } \quad B_{N}(z)=\prod_{n=1}^{15} \frac{\beta e^{i 2 n \pi / 15}-z}{1-\bar{\beta} e^{-i 2 n \pi / 15} z}
$$

which give

$$
\mathcal{L}_{c}\left(K_{B_{M}}\right)=\left\{C_{z}, C_{e^{i \pi / 3} z}, C_{e^{i 2 \pi / 3} z}, C_{-z}, C_{e^{i 4 \pi / 3} z}, C_{e^{i 5 \pi / 3} z}\right\}
$$


and

$$
\mathcal{L}_{c}\left(K_{B_{N}}\right)=\left\{C_{z}, C_{e^{i 2 \pi / 15_{z}}}, C_{e^{i 4 \pi / 15_{z}}}, \ldots, C_{e^{i 28 \pi / 15_{z}}}\right\}
$$

Therefore, by (2.3),

$$
\mathcal{L}_{c}\left(K_{B}\right)=\mathcal{L}_{c}\left(K_{B_{M}}\right) \cap \mathcal{L}_{c}\left(K_{B_{N}}\right)=\left\{C_{z}, C_{e^{i 2 \pi / 3} z}, C_{e^{i 4 \pi / 3} z}\right\}
$$

If we consider the classical situation and start with an analytic symbol $\varphi: \mathbb{D} \longrightarrow \mathbb{D}$, and then try to classify the bounded composition operators on $K_{B}$, we find ourselves in a similar situation as in the proof of Lemma 2.2. As a matter of fact, (2.1) reveals that $\varphi$ has to be a rational function. Hence, in the first place, Lemma 2.2 is applicable and it puts some restrictions on $\varphi$. Secondly, the linear function $\varphi(z)=a z+b$ maps $\mathbb{D}$ into itself if and only if $|a|+|b| \leq 1$ and $|b|<1$. Hence, $\varphi$ maps $\mathbb{D}$ into itself provided that $b=0$. Therefore, we obtain the following special case.

COROLlary 2.4. Let $B$ be a finite Blaschke product with $B(0) \neq 0$, and with at least two distinct zeros, and let $\varphi: \mathbb{D} \longrightarrow \mathbb{D}$ be analytic. Then $C_{\varphi}$ maps $K_{B}$ into itself if and only if the following conditions hold:

(i) $\varphi(z)=a z$ with $a \in \mathbb{T}$.

(ii) $a$ is of finite order, i.e. there is an integer $n \geq 1$ such that $a^{n}=1$.

(iii) If $\lambda$ is a zero of $B$, then so is a $\lambda$.

(iv) The zeros $\lambda, a \lambda, \ldots, a^{n-1} \lambda$ have the same order.

More explicitly, if $a=e^{i 2 \pi / n}, n \geq 2$, and

$$
B(z)=\prod_{j=1}^{m}\left(\prod_{k=1}^{n} \frac{a^{k} \lambda_{j}-z}{1-\overline{a^{k} \lambda_{j}} z}\right)^{m_{j}}, \quad\left(\lambda_{1}, \ldots, \lambda_{m} \in \mathbb{D} \backslash\{0\}\right),
$$

then

$$
\mathcal{L}_{c}\left(K_{B}\right)=\left\{C_{z}, C_{a z}, \ldots, C_{a^{n-1} z}\right\}
$$

Otherwise, in the setting of this case, the only operator in $\mathcal{L}_{c}\left(K_{B}\right)$ whose symbol maps $\mathbb{D}$ into itself is the identity mapping. However, $\mathcal{L}_{c}\left(K_{B}\right)$ might still have other elements. For example, if $B$ is the finite Blaschke product with simple zeros,

$$
\lambda_{1}=\frac{1}{2}, \quad \lambda_{2}=\frac{-1-2 i}{5}, \quad \lambda_{3}=\frac{-3+i}{10}, \quad \lambda_{4}=\frac{i}{3},
$$

then $\varphi(z)=i z+i$ fulfills the requirements of Lemma 2.2. Since $\varphi^{[2]}(z)=-z+i-1$ and $\varphi^{[3]}(z)=-i z-1$ and $\varphi^{[4]}(z)=z$, we deduce that

$$
\mathcal{L}_{c}\left(K_{B}\right)=\left\{C_{i z+i}, C_{-z+i-1}, C_{-i z-1}, C_{z}\right\} .
$$

However, the only symbol that maps $\mathbb{D}$ into itself is $\varphi(z)=z$.

2.3. $\mathcal{Z}(B)=\{0, \lambda\}, B^{\prime}(0) \neq 0$. In this section, we consider the Blaschke products that have a simple zero at the origin, and another (may be multiple) zero at $\lambda \neq 0$. Since a simple zero at the origin creates a constant term in $K_{B}$, the treatment of these types of Blaschke products is a bit different. 
Theorem 2.5. Let

$$
B(z)=z\left(\frac{\lambda-z}{1-\bar{\lambda} z}\right)^{n}, \quad(\lambda \neq 0, n \geq 1)
$$

Then

$$
\mathcal{L}_{c}\left(K_{B}\right)=\left\{C_{\frac{a z+b}{c z+1}}: a \in \mathbb{C}, b \in \mathbb{C} \backslash\{1 / \bar{\lambda}\}, c=(a-1) \bar{\lambda}+b \bar{\lambda}^{2}\right\} .
$$

Proof. The proof has the same flavour as the proof of Theorem 2.1. Let $C_{\varphi} \in$ $\mathcal{L}_{c}\left(K_{B}\right)$. Then, by (1.3), there are some constants $c_{k}$ such that

$$
\left(C_{\varphi} k_{\lambda}^{n}\right)(z)=\frac{1}{(1-\bar{\lambda} \varphi(z))^{n}}=c_{0}+\sum_{k=1}^{n} \frac{c_{k}}{(1-\bar{\lambda} z)^{k}} .
$$

The first term, $c_{0}$, makes the difference in this situation. This representation shows that $\varphi$ is the Möbius transformation that can be normalised as $\varphi(z)=(a z+b) /(c z+1)$. The rest comes from the identity

$$
\left(C_{\varphi} k_{\lambda}\right)(z)=\frac{1}{1-\bar{\lambda}(a z+b) /(c z+1)}=\frac{\alpha z+\beta}{1-\overline{\left(\frac{\bar{a} \lambda-\bar{c}}{-\bar{b} \lambda+1}\right)} z},
$$

where $\alpha$ and $\beta$ are some constants. This forces

$$
\lambda=\frac{\bar{a} \lambda-\bar{c}}{-\bar{b} \lambda+1} .
$$

Hence, on the one hand we must have $b \neq 1 / \bar{\lambda}$, and on the other hand we obtain the equation for $c$.

Note that if we take $a=1-\bar{\lambda} b$, then we obtain the subclass

$$
\varphi(z)=(1-\bar{\lambda} b) z+b, \quad(b \in \mathbb{C} \backslash\{1 / \bar{\lambda}\}) .
$$

Therefore, comparing with Theorem 2.1, we see that the presence of a zero at the origin in the present situation has enlarged $\mathcal{L}_{c}\left(K_{B}\right)$. Moreover, if we pick any arbitrary $b \in \mathbb{C} \backslash\{1 / \bar{\lambda}\}$, and then choose $a=-b \bar{\lambda}$, we would have $a=b c$, which is a singular case and gives the acceptable symbol $\varphi \equiv b$.

COROLlary 2.6. Let

$$
B(z)=z\left(\frac{\lambda-z}{1-\bar{\lambda} z}\right)^{n}, \quad(\lambda \neq 0, n \geq 1),
$$

and let $\varphi: \mathbb{D} \longrightarrow \mathbb{D}$ be analytic. Then $C_{\varphi}$ maps $K_{B}$ into itself if and only if either $\varphi \equiv b \in \mathbb{D}$, or

$$
\varphi(z)=\frac{a z+b}{c z+1}
$$

where $a \in \mathbb{C}, b \in \mathbb{D}$ and $c=(a-1) \bar{\lambda}+b \bar{\lambda}^{2}$, and the constant $a$ and $b$ are such that

$$
|a-b c|+|c-a \bar{b}| \leq 1-|b|^{2} .
$$


Proof. By Theorem 2.5, we must have $\varphi(z)=(a z+b) /(c z+1)$ with $a \in \mathbb{C}, b \in$ $\mathbb{C} \backslash\{1 / \bar{\lambda}\}$, and $c=(a-1) \bar{\lambda}+b \bar{\lambda}^{2}$. Since $\varphi(0)=b$, in any case $b \in \mathbb{D}$.

If $a=b c$, then this is the degenerate case $\varphi \equiv b \in \mathbb{D}$. Now, suppose that $a \neq b c$. Then $\varphi$ maps $\mathbb{D}$ into itself if and only if so does $\tau_{b} \circ \varphi$, where $\tau_{b}$ is the disc automorphism,

$$
\tau_{b}(z)=\frac{b-z}{1-\bar{b} z} .
$$

A simple calculation shows that

$$
\left(\tau_{b} \circ \varphi\right)(z)=\frac{(b c-a) z}{(c-a \bar{b}) z+\left(1-|b|^{2}\right)} .
$$

By Schwarz's lemma, $\tau_{b} \circ \varphi$ is a self-map of $\mathbb{D}$ if and only if

$$
\left|\frac{(b c-a)}{(c-a \bar{b}) z+\left(1-|b|^{2}\right)}\right| \leq 1, \quad(z \in \mathbb{D}) .
$$

This happens if and only if $|c-a \bar{b}|<1-|b|^{2}$ and $|a-b c| \leq 1-|b|^{2}-|c-a \bar{b}|$. But the second inequality implicitly implies the first one.

2.4. $\mathcal{Z}(B)=\left\{0, \lambda_{1}, \lambda_{2}, \ldots\right\}, B^{\prime}(0) \neq 0$. As the passage from Theorems 2.1 to 2.3 revealed that adding extra zeros alters the structure of $\mathcal{L}_{c}\left(K_{B}\right)$, in this section we treat a similar phenomenon by allowing extra distinct zeros to the Blaschke product considered in Theorem 2.5.

LEMMA 2.7. Let $B$ be a finite Blaschke product with $B(0)=0, B^{\prime}(0) \neq 0$, and assume that $B$ has at least two other distinct zeros. Let $\varphi$ be a rational function, $\varphi(z) \neq z$. Then $C_{\varphi}$ maps $K_{B}$ into itself if and only if

$$
\varphi(z)=\frac{a z+b}{c z+d}
$$

and the following conditions are fulfilled:

(i) We have

$$
-2<\frac{a+d}{\sqrt{a d-b c}}<2
$$

(ii) There is an integer $n>1$ such that $\gamma^{n}=1$, where $\gamma$ is given by

$$
\sqrt{\gamma}+\frac{1}{\sqrt{\gamma}}=\frac{a+d}{\sqrt{a d-b c}} .
$$

(iii) If $\lambda \neq 0$ is a zero of $B$, then so is $\eta(\lambda)$, where $\eta$ is the Möbius transformation,

$$
\eta(z)=\frac{\bar{a} z-\bar{c}}{-\bar{b} z+\bar{a}} .
$$

(iv) The zeros $\lambda, \eta(\lambda), \ldots, \eta^{[n-1]}(\lambda)$ have the same order. 
Proof. The proof is slightly more complicated than the proof of Theorem 2.5. Picking any zero $\lambda_{\ell} \neq 0$, by (1.3), there are some constants $c_{n, k}$ such that

$$
\left(C_{\varphi} k_{\lambda_{\ell}}\right)(z)=\frac{1}{1-\bar{\lambda}_{\ell} \varphi(z)}=c_{0}+\sum_{n=1}^{N} \sum_{k=1}^{m_{n}} \frac{c_{n, k}}{\left(1-\bar{\lambda}_{n} z\right)^{k}} .
$$

Again, the presence of $c_{0}$ gives certain liberty in this situation. If we write $\varphi=P / Q$, where $P$ and $Q$ are polynomials with no common divisor, then we must have

$$
\operatorname{deg} Q \leq N_{0}-1 \quad \text { and } \quad \operatorname{deg} P \leq N_{0}-1,
$$

with $N_{0}=1+m_{1}+\cdots+m_{N}$ being the total number of zeros of $B$. Now the same reasoning reveals that the orders of $P$ and $Q$ can be at most one. In other words, $\varphi$ is the Möbius transformation.

Let $\lambda \neq 0$ be any zero of $B$. Then

$$
\left(C_{\varphi} k_{\lambda}^{\ell}\right)(z)=\frac{1}{(1-\bar{\lambda}(a z+b) /(c z+d))^{\ell}}=\frac{(\alpha z+\beta)^{\ell}}{(1-\overline{\eta(\lambda)} z)^{\ell}},
$$

where $\alpha$ and $\beta$ are some appropriate constants. Hence, we conclude that $\eta(\lambda)$ must also be a zero of $B$ and $\eta(\lambda) \neq 0$. Repeating this process shows that, for each $k \geq 1, \eta^{[k]}(\lambda)$ is a zero of $B$ with the same order as $\lambda$.

Since we are dealing with a finite Blaschke product, there must be an integer $n$ such that $\eta^{[n]}=i d$. Therefore, $\eta$ must be an elliptic transformation of finite order. This happens if and only if the normalised trace of $\eta$ is in the interval $(-2,2)$, i.e.

$$
-2<\frac{\bar{a}+\bar{a}}{\sqrt{\bar{a} \bar{a}-\bar{b} \bar{c}}}<2,
$$

and the multiplier of $\eta$, which is given by the equation

$$
\sqrt{\gamma}+\frac{1}{\sqrt{\gamma}}=\frac{\bar{a}+\bar{a}}{\sqrt{\bar{a} \bar{a}-\bar{b} \bar{c}}}
$$

is of finite order. Clearly, in both equations the bars may be removed.

The following result is now immediate. Note that in the light of Lemma 2.10 in the next section, we did not include the assumption $B^{\prime}(0) \neq 0$. But we highlight that there are more restrictions on $\varphi$ in Lemma 2.10, and thus it is more demanding to have $\mathcal{L}_{c}\left(K_{B}\right) \neq\{i d\}$ in that situation.

THEOREM 2.8. Let $B$ be a finite Blaschke product with $B(0)=0$, and assume that $B$ has at least two other distinct zeros. Then there exists an integer $n \geq 1$ and a Möbius function $\varphi(z)=(a z+b) /(c z+d)$ such that $\varphi^{[n]}(z)=z$ and

$$
\mathcal{L}_{c}\left(K_{B}\right)=\left\{C_{z}, C_{\varphi}, C_{\varphi^{[2]}}, \ldots, C_{\varphi^{[n-1]}}\right\} .
$$

In this situation if we seek for symbols $\varphi: \mathbb{D} \longrightarrow \mathbb{D}$, which induce bounded composition operators on $K_{B}$, we see that on the one hand the conditions of Lemma 2.7 must be fulfilled, and on the other hand, similar to Corollary 2.6, we have the extra 
requirement

$$
|a d-b c|+|c \bar{a}-a \bar{b}| \leq|d|^{2}-|b|^{2} .
$$

2.5. $\mathcal{Z}(B)=\{0, \lambda\}, B^{\prime}(0)=0$. If $B$ has multiple zeros at the origin and just one other (maybe multiple) zero, then $\mathcal{L}_{c}\left(K_{B}\right)$ has a very simple structure.

THEOREM 2.9. Let

$$
B(z)=z^{m}\left(\frac{\lambda-z}{1-\bar{\lambda} z}\right)^{n}, \quad(\lambda \neq 0, m \geq 2, n \geq 1) .
$$

Then

$$
\mathcal{L}_{c}\left(K_{B}\right)=\left\{C_{b}: b \in \mathbb{C}, b \neq 1 / \bar{\lambda}\right\}
$$

Proof. As in the proof of Theorem 2.5, we see that $\varphi$ should be the Möbius transformation. But in this case, $f(z)=z \in K_{B}$, and thus we also have the extra requirement

$$
\varphi(z)=\frac{a z+b}{c z+1} \in K_{B}
$$

Therefore,

$$
c=-\bar{\lambda}=(a-1) \bar{\lambda}+b \bar{\lambda}^{2},
$$

which implies $a=-b \bar{\lambda}$. This is a degenerate case and gives $\varphi \equiv b$.

Note that if $B(z)=\gamma z^{m}$, with $m \geq 2$, then

$$
K_{B}=\operatorname{Span}\left\{1, z, \ldots, z^{m-1}\right\}
$$

and thus any symbol $\varphi(z)=a z+b$, with $a, b \in \mathbb{C}$, produces a bounded composition operator on $K_{B}$. However, if we seek only the symbols $\varphi: \mathbb{D} \longrightarrow \mathbb{D}$, we end up with the same characterisation accompanied by extra conditions

$$
|a|+|b| \leq 1, \quad|b|<1 .
$$

2.6. $\mathcal{Z}(B)=\left\{0, \lambda_{1}, \lambda_{2}, \ldots\right\}, B^{\prime}(0)=0$. This is the last case. If $B$ has multiple zeros at the origin and at least two other (maybe multiple) zeros, then, similar to the case of Lemma 2.7, we might have $\mathcal{L}_{c}\left(K_{B}\right) \neq\{i d\}$. However, we have more restrictions in this case.

Lemma 2.10. Let $B$ be a finite Blaschke product with $B(0)=0$. Suppose that the order of $B$ at the origin is $m_{0} \geq 2$ and that $B$ has at least two other distinct zeros. Let $\varphi$ be a rational function, $\varphi(z) \neq z$. Then $C_{\varphi}$ maps $K_{B}$ into itself if and only if

$$
\varphi(z)=\frac{a z+b}{c z+d}
$$


and the following conditions are fulfilled:

(i)

$$
-2<\frac{a+d}{\sqrt{a d-b c}}<2 .
$$

(ii) $\lambda=-\bar{c} / \bar{a}$ is a zero of order at least $m_{0}-1$.

(iii) There is an integer $n>1$ such that $\gamma^{n}=1$, where $\gamma$ is given by

$$
\sqrt{\gamma}+\frac{1}{\sqrt{\gamma}}=\frac{a+d}{\sqrt{a d-b c}} .
$$

(iv) If $\lambda \neq 0$ is a zero of $B$, then so does $\eta(\lambda)$, where $\eta$ is the Möbius transformation

$$
\eta(z)=\frac{\bar{a} z-\bar{c}}{-\bar{b} z+\bar{a}} .
$$

(v) The zeros $\lambda, \eta(\lambda), \ldots, \eta^{[n-1]}(\lambda)$ have the same order.

Proof. The proof is very similar to the proof of Lemma 2.7. With precisely the same method, we see that $\varphi$ should be the Möbius transformation. But in this case $f(z)=z^{m_{0}-1} \in K_{B}$, and thus we have the extra requirement

$$
\varphi(z)=\left(\frac{a z+b}{c z+d}\right)^{m_{0}-1} \in K_{B} .
$$

Therefore, $\lambda=-\bar{c} / \vec{d}$ is a zero of order of at least $m_{0}-1$ for $B$. The other conditions are established as before.

3. When is $\mathcal{L}_{c}\left(K_{B}\right) \neq\{i d\}$ ?. In the light of Lemma 2.2, we face with the following natural question. Let $\Lambda$ be a finite set, not a singleton, of distinct points in $\mathbb{D} \backslash\{0\}$. Under what conditions there are $a \in \mathbb{T}$ of finite order and $b \in \mathbb{C}$ such that $\Lambda$ is invariant under the transformation

$$
\eta(z)=\frac{\bar{a} z}{1-\bar{b} z} ?
$$

Can we explicitly express $a$ and $b$ in terms of elements of $\Lambda$ ? In the same token, one may pose similar questions for Lemmas 2.7 and 2.10.

The case $\Lambda=\left\{\lambda_{1}, \lambda_{2}\right\}$ is simple. In this situation we must have $a^{2}=1$. Since $a \neq 1$, we conclude $a=-1$. To obtain $b$, we use the relation

$$
\lambda_{2}=\eta\left(\lambda_{1}\right)=\frac{-\lambda_{1}}{1-\bar{b} \lambda_{1}}
$$

and obtain

$$
b=\frac{1}{\bar{\lambda}_{1}}+\frac{1}{\bar{\lambda}_{2}} .
$$


Therefore, for any Blaschke product of the form

$$
B(z)=\left(\frac{\lambda_{1}-z}{1-\bar{\lambda}_{1} z}\right)^{n} \times\left(\frac{\lambda_{2}-z}{1-\bar{\lambda}_{2} z}\right)^{n}, \quad\left(\lambda_{1} \neq \lambda_{2} \neq 0, n \geq 1\right),
$$

we have

$$
\mathcal{L}_{c}\left(K_{B}\right)=\left\{C_{z}, C_{-z+1 / \bar{\lambda}_{1}+1 / \bar{\lambda}_{2}}\right\}
$$

If $\Lambda=\left\{\lambda_{1}, \ldots, \lambda_{n}\right\}$, the situation is not that straightforward. In the first place, the ordering in $\Lambda$ becomes important. Without loss of generality, we may take $a=e^{i 2 \pi / n}$. Secondly, if the permutation $\sigma$ is such that $\lambda_{\sigma(k+1)}=\eta\left(\lambda_{\sigma(k)}\right)$, then we must have

$$
b=\frac{1}{\bar{\lambda}_{\sigma(k)}}-\frac{\bar{a}}{\bar{\lambda}_{\sigma(k+1)}}, \quad(k \geq 1) .
$$

In other words, the combination on the right-hand side must be independent of $k$. Moreover, in the above discussion, we assumed that $\Lambda$ has just one cycle. Generally, this is not necessarily the case. The set $\Lambda$ might need to be divided into smaller subsets, each of these make a cycle for some appropriate $a$ and $b$. For example, consider the Blaschke product (2.4) with $M=N=1$ and let $\Lambda$ be the collection of all its zeros. In this case, $\Lambda$ has seven cycles, each with length three.

4. The image of $C_{\varphi}$. In the last two sections, we characterised the symbol $\varphi$ such that the composition operator $C_{\varphi}$ maps $K_{B}$ into itself. As a natural question, we may fix $B$ and $\varphi$ and then ask about the nature of the image space $C_{\varphi} K_{B}$. Of course, in the light of (1.3), we can say that $C_{\varphi} K_{B}$ precisely consists of the function

$$
f(z)=\sum_{k=0}^{m_{0}-1} c_{0, k} \varphi^{k}(z)+\sum_{n=1}^{N} \sum_{k=1}^{m_{n}} \frac{c_{n, k}}{\left(1-\bar{\lambda}_{n} \varphi(z)\right)^{k}} .
$$

However, this subspace is not necessarily invariant under $S^{*}$. A simple example is obtained by taking $B(z)=\varphi(z)=z^{2}$. In this case, $K_{B}=\operatorname{Span}\{1, z\}$ and $C_{\varphi} K_{B}=$ $\operatorname{Span}\left\{1, z^{2}\right\}$. Note that $z^{2} \in C_{\varphi} K_{B}$, but $z=S^{*} z^{2} \notin C_{\varphi} K_{B}$.

Let us denote by $\left\langle C_{\varphi} K_{B}\right\rangle$ the smallest closed $S^{*}$-invariant subspace of $H^{2}$, which contains $C_{\varphi} K_{B}$. Since $C_{\varphi} K_{B}$ is finite dimensional and $\varphi$ is a rational function, $\left\langle C_{\varphi} K_{B}\right\rangle$ is precisely the collection of all elements

$$
f, \quad S^{*} f, \quad S^{* 2} f, \quad \ldots,
$$

where $f$ runs over $C_{\varphi} K_{B}$. Moreover, the above sequence spans a finite dimensional space, and thus for an appropriate index $N, S^{* n} f$ would be a linear combination of $\left\{f, S^{*} f, \ldots, S^{* N} f\right\}$.

In the following, we treat a special case for which we can provide a simple description for $\left\langle C_{\varphi} K_{B}\right\rangle$. We remind that if $B_{1}$ and $B_{2}$ are the finite Blaschke products, so is $B_{1} \circ B_{2}$. 
THEOREM 4.1. Let $B$ and $\varphi$ be finite Blaschke products with $B(0) \neq 0$ and $\varphi(0)=0$. Then the mapping

$$
\begin{aligned}
C_{\varphi}: K_{B} & \longrightarrow K_{B \circ \varphi} \\
f & \longmapsto f \circ \varphi
\end{aligned}
$$

is well defined. Moreover, $K_{B \circ \varphi}$ is the smallest closed $S^{*}$-invariant subspace of $H^{2}$, which contains $C_{\varphi} K_{B}$.

Proof. For simplicity, suppose $B$ has simple zeros. The proof for the general case is similar. Write

$$
B(z)=\prod_{n=1}^{N} \frac{\lambda_{n}-z}{1-\bar{\lambda}_{n} z} \quad \text { and } \quad \varphi(z)=z \prod_{m=1}^{M} \frac{\mu_{n}-z}{1-\bar{\mu}_{n} z} .
$$

Then $K_{B}$ is the linear combination of $k_{\lambda_{1}}, \ldots, k_{\lambda_{N}}$. Hence, $C_{\varphi} K_{B}$ is a linear combination of

$$
\left(C_{\varphi} k_{\lambda_{n}}\right)(z)=\frac{1}{1-\bar{\lambda}_{n} \varphi(z)}, \quad(1 \leq n \leq N) .
$$

Either by Frostman's theorem [6] or by direct verification, we see that

$$
\frac{\lambda_{n}-\varphi(z)}{1-\bar{\lambda}_{n} \varphi(z)}
$$

is a finite Blaschke product with the same order as $\varphi$. Write

$$
\frac{\lambda_{n}-\varphi(z)}{1-\bar{\lambda}_{n} \varphi(z)}=\gamma \prod_{m=1}^{M+1} \frac{\lambda_{n, m}-z}{1-\bar{\lambda}_{n, m} z} .
$$

Put $z=0$ to deduce $\lambda_{n}=\gamma \prod_{m=1}^{M+1} \lambda_{n, m}$. The roots $\lambda_{n, m}$ are in fact the solutions of equation $\varphi(z)=\lambda_{n}$. Thus, we must also have

$$
\lambda_{n}-\varphi(z)=\gamma \frac{\prod_{m=1}^{M+1}\left(\lambda_{n, m}-z\right)}{\prod_{m=1}^{M}\left(1-\bar{\mu}_{n} z\right)} .
$$

Dividing (4.2) by (4.3) gives

$$
\frac{1}{1-\bar{\lambda}_{n} \varphi(z)}=\frac{\prod_{m=1}^{M}\left(1-\bar{\mu}_{n} z\right)}{\prod_{m=1}^{M+1}\left(1-\bar{\lambda}_{n, m} z\right)} .
$$

By doing partial fraction expansion, there are non-zero constants $c_{n, m}$ such that

$$
f(z)=\frac{1}{1-\bar{\lambda}_{n} \varphi(z)}=\sum_{m=1}^{M+1} \frac{c_{n, m}}{1-\bar{\lambda}_{n, m} z} .
$$

It is easy to verify that

$$
S^{*} k_{\lambda}=\bar{\lambda} k_{\lambda}
$$


Hence, for each $\ell \geq 0$,

$$
\left(S^{* \ell} f\right)(z)=\sum_{m=1}^{M+1} c_{n, m} \frac{\bar{\lambda}_{n, m}^{\ell}}{1-\bar{\lambda}_{n, m} z} .
$$

Using the Vandermond determinants, we see that

$$
\operatorname{Span}\left\{S^{* \ell} f: \ell \geq 0\right\}=\operatorname{Span}\left\{1 /\left(1-\bar{\lambda}_{n, m} z\right): 1 \leq m \leq M+1\right\} .
$$

Therefore,

$$
\left\langle C_{\varphi} K_{B}\right\rangle=\operatorname{Span}\left\{1 /\left(1-\bar{\lambda}_{n, m} z\right): 1 \leq m \leq M+1,1 \leq n \leq N\right\}=K_{B \circ \varphi} .
$$

The assumptions $B(0) \neq 0$ and $\varphi(0)=0$ in Theorem 4.1 are not very essential and can be removed. However, the statement of the theorem becomes more complicated. We presented just one case. The other cases can be easily formulated.

\section{REFERENCES}

1. A. Beurling, On two problems concerning linear transformations in Hilbert space, Acta Math. 81 (1949), 239-255.

2. H. Chen and P. Gauthier, Composition operators of $\mu$-Bloch spaces, Canad. J. Math. 61 (2009), 50-75.

3. C. C. Cowen, Composition operators on $H^{2}$, J. Operator Theory 9 (1983), 77-106.

4. C. C. Cowen, Linear fractional composition operators on $H^{2}$, J. Integral Equ. Operator Theory 11 (1988), 151-160.

5. C. Cowen and B. D. MacCluer, Composition operators on spaces of analytic functions, in Studies in Advanced Mathematics, 1st edn. (CRC Press, Boca Raton, FL, 1995), 117-221.

6. O. Frostman, Sur les produits de Blaschke (French), Kungl. Fysiografiska SŤ/llskapets $i$ Lund Frhandlingar (Proc. Roy. Physiog. Soc. Lund) 12(15) (1942), 169-182. MR 0012127 (6:262e)

7. P. Ghatage, D. Zheng and N. Zorboska, Sampling sets and closed range composition operators on the Bloch space, Proc. Amer. Math. Soc. 133 (2004), 1371-1377.

8. J. Gimémez, R. Malavé and J. C. Ramos Fernàndez, Composition operators on $\mu$-Bloch type spaces, Rend. Circ. Mat. Palermo 59(2) (2010), 107-119. $237-248$.

9. B. MacCluer, Compact composition operators in $H^{p}\left(B_{N}\right)$, Michigan Math. J. 32 (1985),

10. B. D. MacCluer and J. H. Shapiro, Angular derivatives and compact composition operators on the Hardy and Bergman spaces, Canad. Math. J. 38 (1986), 878-906.

11. B. D. MacCluer, X. Zeng and N. Zorboska, Composition operators on small weighted Hardy spaces, Illinois J. Math. 40 (1996), 662-667.

12. T. Needham, Visual complex analysis (Oxford University Press, Oxford, UK, 2002).

13. J. H. Shapiro, Composition operators and classical function theory, in Univeritext: Tracts in Mathematics (Springer-Verlag, 1993).

14. J. H. Shapiro and P. D. Taylor, Compact, nuclear and Hilbert-Schmidt composition operators on $H^{2}$, Indiana Univ. Math. J. 23 (1973), 471-496.

15. M. Tjani, Compact composition operators on Besov spaces, Trans. Amer. Math. Soc. 355(11) (2003), 4683-4698.

16. H. Wulan, D. Zheng and K. Zhu, Compact composition operators on BMOA and the Bloch space, Proc. Amer. Math. Soc. 137 (2009), 3861-3868.

17. N. Zorboska, Composition operators on weighted Dirichlet spaces, Proc. Amer. Math. Soc. 126(7) (1998), 2013-2023. 